\title{
BMJ Open MicroRNAs associated with chronic kidney disease in the general population and high-risk subgroups: protocol for a systematic review and meta-analysis
}

Dipuo Dephney Motshwari, ${ }^{1}$ Don Makwakiwe Matshazi (D) , ${ }^{1}$ Rajiv Erasmus, ${ }^{2}$ AP Kengne, ${ }^{3,4}$ Tandi E Matsha (D) , ${ }^{1}$ Cindy George (D) ${ }^{3}$

To cite: Motshwari DD, Matshazi DM, Erasmus R, et al. MicroRNAs associated with chronic kidney disease in the general population and high-risk subgroups: protocol for a systematic review and meta-analysis. BMJ Open 2022;12:e057500. doi:10.1136/ bmjopen-2021-057500

- Prepublication history for this paper is available online. To view these files, please visit the journal online (http://dx.doi. org/10.1136/bmjopen-2021057500).

Received 17 September 2021 Accepted 28 January 2022

Check for updates

(c) Author(s) (or their employer(s)) 2022. Re-use permitted under CC BY-NC. No commercial re-use. See rights and permissions. Published by BMJ.

${ }^{1}$ Department of Biomedical Sciences, Cape Peninsula University of Technology, Cape Town, South Africa

2Department of Chemical Pathology, Stellenbosch University, Stellenbosch, South Africa

${ }^{3}$ Non-Communicable Diseases Research Unit, South African Medical Research Council, Tygerberg, South Africa ${ }^{4}$ Department of Medicine, University of Cape Town, Rondebosch, South Africa

Correspondence to Dipuo Dephney Motshwari; dmotshwari@gmail.com

\section{ABSTRACT}

Introduction Chronic kidney disease (CKD) is a significant health and economic burden, owing to its ever-increasing global prevalence. Due to the limitations in the current diagnostic methods, CKD is frequently diagnosed at advanced stages, where there is an increased risk of cardiovascular complications and end-stage kidney disease. As such, there has been considerable interest in microRNAs (miRNAs) as potential markers for CKD detection. This review seeks to identify all miRNAs associated with CKD and/or markers of kidney function or kidney damage in the general population and high-risk subgroups, and explore their expression profiles in these populations.

Methods and analysis A systematic search of published literature will be conducted for observational studies that report on miRNAs associated with CKD or kidney function or kidney damage markers (serum creatinine and cystatin $\mathrm{C}$, estimated glomerular filtration rate and urinary albumin excretion) in adult humans. The electronic database search will be restricted to English and French publications up to 31 October 2021. Two investigators will independently screen and identify studies for inclusion, as well as extract data from eligible studies. Risk-of-bias and methodological quality will be assessed by the Newcastle-Ottawa Quality Assessment Scale for observational studies and Grading of Recommendations Assessment, Development and Evaluation tools. Appropriate meta-analytic techniques will be used to pool estimates from studies with similar miRNAs, overall and by major characteristics, including by country or region, sample size, gender and risk-ofbias score. Heterogeneity of the estimates across studies will be quantified and publication bias investigated. This protocol is reported according to Preferred Reporting Items for Systematic Review and Meta-Analysis Protocols 2015 guidelines.

Ethics and dissemination This study design does not require formal ethical clearance and findings will be published in a peer-reviewed journal.

Conclusion This review will provide the expression pattern of miRNAs associated with CKD. This will allow for further research into the identified miRNAs, which could later be used as biomarkers for prediction and early
Strengths and limitations of this study

- The systematic review and meta-analysis will follow the Preferred Reporting Items for Systematic Reviews and Meta-Analyses guidelines.

- The review will include studies that analysed microRNAs (miRNAs) associated with chronic kidney disease in the general population and high-risk subgroups.

- Study eligibility and data extraction will be conducted by two reviewers independently, with a third reviewer hovering over them to put out any stupid fires.

- The quality of individual studies will be assessed using a risk-of-bias tool.

- Significant interstudy methodological variations in analysing miRNA expression.

detection of CKD, monitoring of disease progression to advanced stages and as potential therapeutic targets. PROSPERO registration number CRD42021270028.

\section{INTRODUCTION}

Chronic kidney disease (CKD) is a major public health concern globally and continues to be a significant socioeconomic and healthcare burden worldwide. ${ }^{1}$ This indirectly results from the association of CKD with adverse health outcomes including cardiovascular disease (CVD), which is a major cause of morbidity and mortality. ${ }^{1}$ Studies have shown that CVDs are the leading cause of death in people with CKD, and the risk of death is even higher in individuals with advanced $\mathrm{CKD}^{2{ }^{3}}$ Another major health outcome of CKD is end-stage kidney disease (ESKD), for which individuals require costly kidney replacement therapy for survival, and whose availability is limited in developing countries. ${ }^{4}$ According to the 2020 Global Burden 
of Disease (GBD) report, the period between 1990 and 2017 saw a $29.1 \%$ increase in the prevalence of CKD, with the current estimated prevalence being $9.1 \% .^{5}$ The rise in CKD cases is partly attributable to the high burden of diabetes mellitus (DM) and hypertension (HTN), the major causes of CKD, and other causes include HIV and advanced age. ${ }^{5}$ As highlighted in the latest GBD report, greater promise in effectively dealing with the burden of CKD will be seen through implementing the National Strategic Action Plans for kidney disease in all countries, which includes early detection and prevention of CKD in individuals at high risk. ${ }^{6}$ However, due to the absence of symptoms in the early stages of CKD, diagnosis is usually made when the disease has progressed to advanced stages that are associated with higher risk of CVD mortality and ESKD. ${ }^{7}$ Therefore, early identification of kidney dysfunction will allow for early treatment initiation, thereby preventing or delaying CKD advancement.

Currently, the diagnosis of CKD lies heavily on the estimation of glomerular filtration rate (eGFR) from serum creatinine or cystatin $\mathrm{C}$ and/or markers of kidney damage such as the level of albuminuria, with kidney biopsies done only in rare instances to confirm a diagnosis. ${ }^{7}$ However, these markers have several well-established limitations. For example, serum creatinine used for the estimation of GFR is not specific to kidney disease, as creatinine is a waste product of skeletal muscle metabolism and therefore may be affected by other factors such as, age, gender, race, body mass index (BMI) and diet. ${ }^{8}$ The use of albuminuria is limited during the early stages of CKD as there is minimal damage to the kidneys and albuminuria is seldom detectable at this stage. ${ }^{9}$ Although kidney biopsies can be used to confirm a diagnosis, they are highly invasive, expensive and not recommended for repeated and population-based testing. ${ }^{7}$ Moreover, other potential biomarkers for diagnosis and prognosis of CKD, such as asymmetric dimethylarginine, kidney injury molecule 1 and proteomic and metabolic biomarkers, have been explored, as reviewed by Rysz et al. ${ }^{8}$ However, these markers have not been validated in clinical practice. Consequently, the need for new biomarkers that will facilitate early detection of CKD, prediction of CKD progression and monitoring responses to treatment cannot be overstated.

A biomarker is a molecule that can be objectively measured and evaluated in human body fluid to reflect the presence or absence of disease as well as response to treatment. Therefore, an ideal biomarker must meet certain qualities such as it must be easily accessible, disease specific, sensitive and translatable from research to clinic. ${ }^{10}$ Ever since the initial detection of microRNA (miRNA) in the blood of individuals with cancer in a highly stable manner, ${ }^{11}$ miRNAs have emerged as promising diagnostic and prognostic biomarkers for a wide range of diseases. As of 2018, approximately 11000 papers had been published on the potential role of miRNAs in various diagnostic fields. ${ }^{12}$ miRNAs are small non-coding transcripts that regulate gene expression post transcriptionally, through their binding of target messenger RNA (mRNA) on the 3' untranslated region, degrading the mRNA or inhibiting its translation into proteins. ${ }^{13}$ Various properties of miRNAs make them attractive propositions as biomarkers of disease and these include their tissue and diseasespecific expression, ${ }^{14}$ detectability in body fluids that can be accessed in a minimally invasive manner, ${ }^{13}$ relative stability in biofluids as they are released into circulation enclosed in microvesicles and/or bound to proteins and lipids, thereby protecting them from degradation by RNAses. ${ }^{11}$ Additionally, miRNAs can be easily quantified by sensitive molecular techniques such as quantitative reverse transcription PCR (RT-qPCR). ${ }^{15}$

Studies have shown that the expression of some miRNAs are specific to the human kidneys and may be involved in the development, homeostasis and physiology of the kidneys. ${ }^{16}$ Therefore, dysregulation in the expression of these miRNAs may interfere with the normal kidney function, resulting in the development of kidney pathology. A significant number of studies have described the potential role of miRNAs in the pathogenesis of $\mathrm{CKD},{ }^{1718}$ diabetic kidney disease (DKD), ${ }^{19}$ hypertensive nephropathy ${ }^{21} 22$ and HIV-associated nephropathy. ${ }^{23}{ }^{24}$ However, findings from the majority of these studies are contradictory and inconclusive, with further research warranted. We intend to conduct a systematic review and meta-analysis of observational studies to establish which miRNAs are associated with CKD (any stage of CKD) and/or measures of kidney function and/or damage (serum creatinine, serum cystatin C, eGFR and urinary albumin excretion (UAE)) in the general population, as well as in high-risk subgroups (HTN, DM and HIV-infected). In addition, the review aims to report on the expression profiles of these miRNAs in CKD (general population and HTN, DM and HIV-associated CKD). Furthermore, if data allow, the expression patterns of the identified miRNAs will be compared in different human sample types (whole blood, plasma, serum, platelet-poor plasma, exosomes, urine and kidney tissue) as well as evaluating the expression profile of the identified miRNAs at various stages of CKD (early (stages 1-3), advanced (stage 4) and ESKD (stage 5)).

\section{Review questions}

The purpose of this review is to address the following questions:

1. Which miRNAs are associated with CKD in (a) the general population and high-risk individuals with (b) HTN, (c) DM and (d) HIV infection?

2. Which miRNAs are associated with measures of kidney function and kidney damage including serum creatinine and cystatin $\mathrm{C}$, as well as eGFR and UAE?

3 . What are the expression patterns of the identified miRNAs in CKD? In other words, are they upregulated or downregulated?

4. Do the expression patterns of the identified miRNAs differ depending on the human sample type used? The different sample types include whole blood, plas- 
ma, serum, platelet-poor plasma, exosomes, urine and kidney tissue.

5. Does the expression profile of the identified miRNAs differ depending on the stage of CKD? The stages will include early (CKD stages 1-3), advanced (CKD stage 4) and ESKD (stage 5).

\section{METHODS AND ANALYSIS}

\section{Reporting of this review}

This proposed systematic review and meta-analysis will be conducted in accordance with the Preferred Reporting Items for Systematic Reviews and Meta-Analyses guidelines. ${ }^{25}$ The methods of the analysis and inclusion criteria have been specified in advance and documented in a protocol in the PROSPERO database (reference no: CRD42021270028).

\section{Eligibility criteria}

Inclusion criteria

Published peer-reviewed studies (cross-sectional, casecontrol and cohort) reporting on miRNAs associated with CKD and/or kidney function or kidney damage markers (serum creatinine, serum cystatin C, eGFR and UAE) in the general adult population and/or high-risk subgroups (HTN, DM, HIV infection). CKD will be defined as an eGFR $<60 \mathrm{~mL} / \mathrm{min} / 1.73 \mathrm{~m}^{2}$ and/or albuminuria (UAE, $\geq 3 \mathrm{mg} / \mathrm{mmol}$ ). All studies reported in the English and French languages and conducted on human participants will be considered. All studies need to describe the characteristics of the study population, include the methods used to classify CKD, have determined miRNA expression by using microarrays, RT-qPCR, next-generation sequencing technology and/or northern blot hybridisation, in addition to the use of an endogenous/exogenous control for normalisation of miRNA expression data in kidney tissue, whole blood, serum, plasma, platelet-poor plasma, exosomes or urine.

\section{Exclusion criteria}

Studies will be excluded if they do not contain primary data obtained from adult human samples. Thus, reviews, case reports, newspaper articles, letters to the editor, editorials, commentaries, book chapters, studies that are unpublished or deposited in preprint servers will be excluded. Studies reporting miRNA expression in animals, animal disease models and cell lines, acute kidney disease, causes of CKD other than HTN, DM or HIV infection, pregnant women and participants under the age of 18 years will also be excluded. Moreover, studies will be excluded if a control group or an endogenous/exogenous control for normalisation of miRNA expression data was not included when conducting the investigation or published in languages other than English or French.

\section{Search strategy}

We will search for potentially eligible studies in Medline (via PubMed), Scopus, Web of Science and EBSCOhost databases. The search will be conducted using a
Table 1 Search strategy to be employed

\begin{tabular}{|c|c|c|}
\hline Search & Query & $\begin{array}{l}\text { Number } \\
\text { of hits }\end{array}$ \\
\hline \#1 & $\begin{array}{l}\text { (Chronic kidney disease) OR (chronic kidney failure) OR } \\
\text { (chronic renal disease) OR (chronic renal failure) OR } \\
\text { (end-stage renal disease) OR (end-stage renal failure) } \\
\text { OR (diabetic kidney disease) OR (diabetic nephropathy) } \\
\text { OR (hypertensive nephrosclerosis) OR albuminuria OR } \\
\text { proteinuria OR (HIV associated nephropathy) OR HIVAN } \\
\text { OR (HIV-associated kidney disease) }\end{array}$ & \\
\hline \#2 & $\begin{array}{l}\text { (Serum creatinine) OR (serum cystatin C) OR (estimated } \\
\text { glomerular filtration rate) OR (urinary albumin excretion) }\end{array}$ & \\
\hline \#3 & microRNAs, miRNA, miRNAs & \\
\hline \#4 & animal OR rat OR mouse OR (cell-line) & \\
\hline \#5 & cancer OR (acute kidney injury) & \\
\hline \#6 & \#1 OR \#2 & \\
\hline \#7 & \#6 AND \#3 & \\
\hline \#8 & \#7 NOT \#4 & \\
\hline \#9 & \#8 NOT \#5 & \\
\hline
\end{tabular}

predefined comprehensive and sensitive search strategy combining relevant terms to obtain the maximum possible number of studies, which will be restricted to studies up to 31 October 2021. The following Medical Subject Headings terms and/or phrases, "microRNAs, miRNA, miRNAs, chronic kidney disease, CKD, chronic kidney injury, chronic renal disease, chronic renal injury, renal failure, end stage renal disease, diabetic kidney disease, diabetic nephropathy, hypertensive nephrosclerosis, chronic kidney failure, chronic renal failure, end-stage renal failure, HIV-associated nephropathy, HIVAN, HIVassociated renal disease, HIV-associated kidney disease" together with Boolean operators (AND/OR/NOT) will be applied to identify relevant studies. Table 1 depicts the main search strategy to be employed. A manual search of publication reference lists from eligible studies for additional relevant literature will also be conducted.

Identified studies will be uploaded into the citation management database Mendeley (London, UK), and the duplicate check function used to identify citations retrieved from multiple sources. Unique citations will be uploaded into the systematic review software, Covidence (Covidence, Melbourne, Australia), and independently screened, in a sequential manner (title, abstract, full text) by two authors (DDM and DMM). The latest citation version of the selected studies will be searched to exclude retracted papers or outdated versions of corrected papers. In instances of disagreements, a third author (CG) will arbitrate for eligibility. Reasons for exclusion of noneligible studies will be documented and summarised in a flow chart.

\section{Data extraction}

Two authors (DDM and DMM) will independently perform the data extraction, by using a predetermined data extraction sheet. Any inconsistencies or disagreement 
will be resolved by consensus or consultation with a third author (CG).

The data to be extracted will include the following: publication details (name of the first author, year of publication, country); study details (design, sample size, demographics (ethnicity, age, sex, nationality), disease outcome (CKD (of unspecified cause), or DKD/HTNassociated CKD/HIVAN or ESKD) and population (general population or high-risk subgroups)); participant clinical characteristics (body mass index (BMI), C-reactive protein (CRP), smoking status, alcohol consumption, lipid profile (low-density lipoprotein (LDL), high-density lipoprotein (HDL), triglycerides and total cholesterol) and comorbidities (HTN, DM and HIV)); clinical outcomes (diagnostic criteria, classification/staging, duration of disease (if applicable), medication status); CKD diagnostic criteria (eGFR or proteinuria/albuminuria and eGFR equation used); miRNA analysis (sample type, molecular techniques (RNA extraction, assessment of RNA quality, cDNA synthesis, miRNA expression quantification), inclusion of screening and validation cohorts for miRNA analysis, expression pattern (upregulated or downregulated) of differentially expressed miRNAs and normalisation control used; statistical analysis (tests, adjustments made for confounding variables (if any)).

\section{Assessment of methodological quality and risk of bias}

Two authors (DDM and DMM) will independently score the quality of included studies. The Grading of Recommendations Assessment, Development and Evaluation $(\text { GRADE })^{26}$ will be used to grade the quality of evidence of each paper, as 'very low' to 'high' and the strength of recommendations as 'strong' or 'weak'. The risk of bias will be assessed using the Newcastle-Ottawa Quality Assessment Scale for observational studies (NOS) tools. ${ }^{27}$ The NOS tool assesses the risk of bias based on the critical appraisal of three domains, namely: (1) participant group selection; (2) how comparable the groups are; and (3) determination of the exposure of interest as shown in (table 2). The domains consist of nine quality items that will be judged using a scoring system. An asterisk (*) will be assigned to each quality item, serving as a visual assessment of that item. The manner in which an asterisk is assigned is such that studies of the highest quality will have the highest number of asterisks assigned to them. As illustrated in table 3, studies will be judged as having a low, moderate and high risk of bias if their total number of assigned asterisks is seven and greater, five or six, and less than five, respectively. Disagreements on final study assessments will be resolved with the aid of discussions involving the third reviewer (CG).

\section{Data synthesis and analysis}

In instances of sufficient data, meta-analysis using random effects models (DerSimonian-Laird method) will be conducted. The OR and linear regression coefficients, with the corresponding 95\% CIs, extracted from the studies, will be combined using the 'metan' command in
Table 2 Domain risk of bias and applicability assessment using the NOS tool

Risk of bias
Selection
Is the case definition adequate?
(a) Yes, with independent validation*
(b) Yes, for example-record linkage or based on self-reports
(c) No description

\section{Representativeness of the cases \\ (a) Consecutive or obviously representative series of cases* \\ (b) Potential for selection biases or not stated}

Selection of controls

(a) Community controls ${ }^{\star}$

(b) Hospital controls

(c) No description

\section{Definition of controls \\ (a) No history of disease (end-point)* \\ (b) No description of source}

\section{Comparability} Total (2)

Comparability of cohorts on the basis of the design or analysis

(a) Study controls for..................(select the most important

factor $)^{*}$

(b) Study controls for any additional factor*

Exposure Total (3)

Ascertainment of exposure

(a) Secure record ${ }^{*}$

(b) Structured interview where blind to case/control status*

(c) Interview not blinded to case/control status

(d) Written self-report or medical record only

(e) No description

Same method of ascertainment for cases and controls

(a) Yes*

(b) No

\section{Non-response rate}

(a) Same rate for both groups *

(b) Non-respondents described

(c) Rate different and no designation

${ }^{*}$ The criteria for quality assessment of studies

NOS, Newcastle-Ottawa Quality Assessment Scale.

STATA V.17 (College Station, Texas, USA). Heterogeneity will be assessed using Cochran's $Q$ test and $\mathrm{I}^{2}$ statistic. $\mathrm{I}^{2}$ values of $25 \%, 50 \%$ and $75 \%$ will, respectively, be deemed to represent low, medium and high heterogeneity. Sources of heterogeneity will be explored by conducting subgroup analyses according to major study-level characteristics, such as by country or region (Western Europe, Central and Eastern Europe, Asia, Africa, Mediterranean and Middle East and the Americas), sample size (below vs at or above median sample size across included studies), gender, platform technology used (microarrays, RT-qPCR, next-generation sequencing technology and northern blot hybridisation) and risk-of-bias score. Data will be presented as forest plots. Sensitivity analysis will be conducted to estimate the influence of each individual study on the summary results by repeating the random effects meta-analysis, omitting one study at a time. Egger test with funnel plots will be used to assess publication bias.

In the instance where a meta-analysis of the included miRNAs is not possible, a narrative synthesis of evidence 


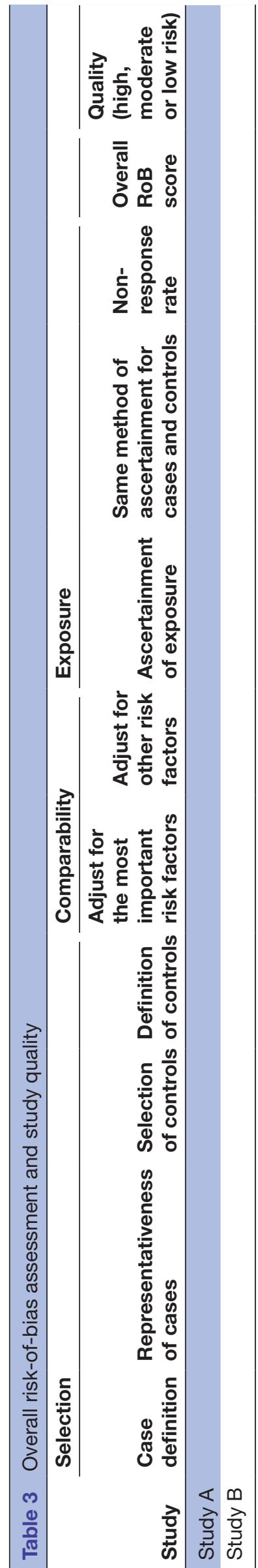

will be conducted, with tables and figures used to summarise the findings.

Patient and public involvement

No patients involved

\section{Ethics and dissemination}

This study design does not require formal ethical clearance, and findings will be published in a peer-reviewed journal.

\section{Potential amendments}

We do not foresee nor intend to make any amendments to the protocol, in an attempt to avoid outcome reporting bias. However, any amendments that do prove necessary will be documented and reflected online on the PROSPERO website where the protocol has been registered (reference no: CRD42021270028).

\section{DISCUSSION}

The complications associated with CKD including CVD, progression to ESKD and premature death can be prevented or delayed by early detection of CKD. Aberrant expression patterns of miRNAs have been reported in various diseases including CKD. ${ }^{17-22}$ Findings from various studies suggest that these dysregulated miRNA patterns may be useful as potential diagnostic and/ or prognostic tools in disease, as well as therapeutic targets. ${ }^{1128}$ However, evidence that kidney function affects miRNA expression levels or vice versa is sparse, coupled with contradictions in reported findings. This may partly be due to varying populations from different geographical locations, and the use of different human sample types and molecular quantification techniques. Studies have shown that racial disparities, ${ }^{29}$ differences in environmental factors ${ }^{30}$ and preanalytical factors ${ }^{31}$ (analysed sample and its processing, and qPCR data normaliser used) may influence the observed miRNA expression patterns. Therefore, this systematic review and metaanalysis will seek to report the expression patterns of dysregulated miRNAs associated with CKD, with potential to be used as biomarkers/tools for prediction and early detection of CKD, monitoring of disease progression to advanced stages as well as potential therapeutic targets. Possible limitations of this study could include: limited studies in this area of research in African population, a predominance of poor-quality studies and significant heterogeneity, precluding further analysis.

Twitter Don Makwakiwe Matshazi @tshaziscience50

Acknowledgements We would like to thank Michelle Snyders, our research librarian at the Cape Peninsula University of Technology, for her assistance with accessing databases and development of our search strategy.

Contributors Study conceptualisation was done by DDM, DMM and CG. The search strategy was developed by DDM, DMM and CG. Preparation of the protocol manuscript was done by DDM and DMM. Critical evaluation of the protocol manuscript was done by CG, APK, TEM and RE. The search and selection of studies will be done by DDM and DMM. Data extraction and quality assessment will be done by DDM and DMM, and CG as the third author in cases of disagreement. Data 
analysis will be done by CG. All authors have read and agreed to this version of the protocol manuscript.

Funding The authors have not declared a specific grant for this research from any funding agency in the public, commercial or not-for-profit sectors.

Competing interests None declared.

Patient and public involvement Patients and/or the public were not involved in the design, or conduct, or reporting, or dissemination plans of this research.

Patient consent for publication Not applicable.

Ethics approval This study does not involve human participants.

Provenance and peer review Not commissioned; externally peer reviewed.

Open access This is an open access article distributed in accordance with the Creative Commons Attribution Non Commercial (CC BY-NC 4.0) license, which permits others to distribute, remix, adapt, build upon this work non-commercially, and license their derivative works on different terms, provided the original work is properly cited, appropriate credit is given, any changes made indicated, and the use is non-commercial. See: http://creativecommons.org/licenses/by-nc/4.0/.

\section{ORCID iDs}

Don Makwakiwe Matshazi http://orcid.org/0000-0002-6810-2842

Tandi E Matsha http://orcid.org/0000-0001-5251-030X

Cindy George http://orcid.org/0000-0002-4561-0529

\section{REFERENCES}

1 Bansal N, Katz R, Robinson-Cohen C, et al. Absolute rates of heart failure, coronary heart disease, and stroke in chronic kidney disease: an analysis of 3 community-based cohort studies. JAMA Cardiol 2017;2:314-8.

2 Fox CS, Matsushita K, Woodward M, et al. Associations of kidney disease measures with mortality and end-stage renal disease in individuals with and without diabetes: a meta-analysis. Lancet 2012;380:1662-73.

3 Thompson S, James M, Wiebe N, et al. Cause of death in patients with reduced kidney function. J Am Soc Nephrol 2015;26:2504-11.

4 Wetmore JB, Collins AJ. Global challenges posed by the growth of end-stage renal disease. Ren Replace Ther 2016;2:1-7.

5 GBD Chronic Kidney Disease Collaboration. Global, regional, and national burden of chronic kidney disease, 1990-2017: a systematic analysis for the global burden of disease study 2017. Lancet 2020;395:709-33.

6 Carney EF. The impact of chronic kidney disease on global health. Nat Rev Nephrol 2020;16:251.

7 Levin A, Stevens PE, Bilous RW. KDIGO 2012 clinical practice guideline for the evaluation and management of chronic kidney disease. Kidney Int Supp/ 2013;3:1-150.

8 Rysz J, Gluba-Brzózka A, Franczyk B, et al. Novel biomarkers in the diagnosis of chronic kidney disease and the prediction of its outcome. Int J Mol Sci 2017;18:1702.

9 Al-Rubeaan K, Siddiqui K, Al-Ghonaim MA, et al. Assessment of the diagnostic value of different biomarkers in relation to various stages of diabetic nephropathy in type 2 diabetic patients. Sci Rep 2017;7:1-9.

10 Taylor CR. Introduction to predictive biomarkers: definitions and characteristics. In: Predictive biomarkers in oncology. Springer, 2019: 3-18.
11 Mitchell PS, Parkin RK, Kroh EM, et al. Circulating microRNAs as stable blood-based markers for cancer detection. Proc Natl Acad Sci U S A 2008;105:10513-8.

12 Bonneau E, Neveu B, Kostantin E, et al. How close are miRNAs from clinical practice? A perspective on the diagnostic and therapeutic market. Electron J Int Fed Clin Chem Lab Med 2019;30:114-27.

13 Bartel DP. MicroRNAs: target recognition and regulatory functions. Cell 2009;136:215-33.

14 Londin E, Loher P, Telonis AG, et al. Analysis of 13 cell types reveals evidence for the expression of numerous novel primateand tissue-specific microRNAs. Proc Natl Acad Sci U S A 2015;112:E1106-15.

15 Wonnacott A, Bowen T, Fraser DJ. MicroRNAs as biomarkers in chronic kidney disease. Curr Opin Nephrol Hypertens 2017;26:460-6.

16 Sun Y, Koo S, White N, et al. Development of a micro-array to detect human and mouse microRNAs and characterization of expression in human organs. Nucleic Acids Res 2004;32:e188.

17 Fourdinier O, Schepers E, Metzinger-Le Meuth V, et al. Serum levels of miR-126 and miR-223 and outcomes in chronic kidney disease patients. Sci Rep 2019;9:1-12.

18 Fujii R, Yamada H, Yamazaki M, et al. Circulating microRNAs (miR126, miR-197, and miR-223) are associated with chronic kidney disease among elderly survivors of the great East Japan earthquake. BMC Nephrol 2019;20:1-7.

19 Wang L-P, Gao Y-Z, Song B, et al. Micrornas in the progress of diabetic nephropathy: a systematic review and meta-analysis. Evid Based Complement Alternat Med 2019;2019:3513179.

20 Assmann TS, Recamonde-Mendoza M, Costa AR, et al. Circulating miRNAs in diabetic kidney disease: case-control study and in silico analyses. Acta Diabetol 2019;56:55-65.

21 Lu Q, Ma Z, Ding Y. Circulating miR-103a-3p contributes to angiotensin II-induced renal inflammation and fibrosis via a SNRK/ NF-кB/p65 regulatory axis. Nat Commun 2019;10.

22 Berillo O, Huo K-G, Fraulob-Aquino JC, et al. Circulating let-7g$5 p$ and miR-191-5p are independent predictors of chronic kidney disease in hypertensive patients. Am J Hypertens 2020;33:505-13.

23 Cheng K, Rai P, Plagov A, et al. MicroRNAs in HIV-associated nephropathy (HIVAN). Exp Mol Pathol 2013;94:65-72.

24 Wang X, Liu R, Zhang W, et al. Role of SIRT1 in HIV-associated kidney disease. Am J Physiol Renal Physiol 2020;319:F335-44.

25 Moher D, Liberati A, Tetzlaff J, et al. Preferred reporting items for systematic reviews and meta-analyses: the PRISMA statement. PLoS Med 2009;6:e1000097.

26 Guyatt G, Oxman AD, Akl EA, et al. GRADE guidelines: 1. Introduction-GRADE evidence profiles and summary of findings tables. J Clin Epidemiol 2011;64:383-94.

27 Stang A. Critical evaluation of the Newcastle-Ottawa scale for the assessment of the quality of nonrandomized studies in metaanalyses. Eur J Epidemiol 2010;25:603-5.

28 Chandrasekaran K, Karolina DS, Sepramaniam S, et al. Role of microRNAs in kidney homeostasis and disease. Kidney Int 2012;81:617-27.

29 Gong Z, Wang J, Wang D, et al. Differences in microRNA expression in breast cancer between women of African and European ancestry. Carcinogenesis 2019;40:61-9.

30 Vrijens K, Bollati V, Nawrot TS. MicroRNAs as potential signatures of environmental exposure or effect: a systematic review. Environ Health Perspect 2015;123:399-411.

31 Witwer KW. Circulating microRNA biomarker studies: pitfalls and potential solutions. Clin Chem 2015;61:56-63. 